Article

\title{
TIME AND MODE OF EPIDEMIC HCV-2 SUBTYPES DISPERSAL IN EUROPE: PHYLODYNAMICS OF HCV- 2C IN ITALY AND ALBANIA
}

\author{
Erika Ebranati 1,2, Alessandro Mancon ${ }^{3}$, Martina Airoldi ${ }^{1}$, Silvia Renica ${ }^{1}$, Renata Shkjezi ${ }^{4}$, Pranvera \\ Dragusha ${ }^{4}$, Carla Della Ventura ${ }^{1}$, Annarita Ciccaglione ${ }^{5}$, Massimo Ciccozzi ${ }^{6}$, Silvia Bino ${ }^{7}$, Elisabetta \\ Tanzi ${ }^{8}$, Valeria Micheli ${ }^{3}$, Elisabetta Riva ${ }^{9}$, Massimo Galli 1,2, Gianguglielmo Zehender ${ }^{1,2, *}$. \\ ${ }^{1}$ Department of Biomedical and Clinical Sciences "L. Sacco", University of Milan, Milano, Italy; \\ erika.ebranati@gmail.com (E.E.); martina.airoldi@uni-wuerzburg.de (M.A.); silvia.renica@unimi.it (S.R.); \\ carla.dellaventura@gmail.com (C.D.V.); massimo.galli@unimi.it (M.G.); \\ ${ }^{2}$ CRC-Coordinated Research Center "EpiSoMI", University of Milan, Milano, Italy \\ ${ }^{3}$ Unit of Microbiology, Hospital Sacco of Milan, Milan, Italy; alessandro.mancon@live.com (A.M.); \\ valeria.micheli@asst-fbf-sacco.it (V.M.) \\ ${ }^{4}$ Catholic University "Our Lady of the Good Counsel", Faculty of Medicine and Surgery, Tirana, Albania; \\ renata_sh@libero.it (R.S.); p.dragusha@unizkm.al (P.D.) \\ ${ }^{5}$ Viral Hepatitis Unit, Department of Infectious, Parasitic and Immune-Mediated Diseases, Istituto Superiore di Sanità, \\ Rome, Italy; annarita.ciccaglione@iss.it (A.C.) \\ ${ }^{6}$ Unit of Medical Statistics and Molecular Epidemiology, University Campus Bio-Medico of Rome, Italy; \\ m.ciccozzi@unicampus.it (M.C.); \\ ${ }^{7}$ National Institute of Health, Tirana, Albania ;silvia.bino@gmail.com (S.B.) \\ ${ }^{8}$ Department of Biomedical Sciences for the Health, University of Milan, Milan, Italy; elisabetta.tanzi@unimi.it (E.T.) \\ ${ }^{9}$ Laboratory of Virology, Campus Bio-Medico University, Rome, Italy; e.riva@unicampus.it (E.R.) \\ *Correspondence: gianguglielmo.zehender@unimi.it; +02-503-19770 (G.Z.)
}

\begin{abstract}
Newly characterising 245 Italian and Albanian HCV-2 NS5B sequences collected between 2001 and 2016 was used to reconstruct the origin and dispersion pathways of HCV-2c. The tree of a subset of these sequences aligned with 247 publicly available sequences was reconstructed in spatio-temporal scale using the Bayesian approach, and the effective replication number (Re) was estimated using the birth-death model. Our findings show that HCV-2c was the most prevalent subtype in Italy and Albania, and that GT2 originated in Guinea Bissau in the XVI century and spread to Europe in the XX century. The HCV-2c subtype had two internal nodes respectively dating back to the 1930s and 1950s having as most probable locations Ghana and Italy, respectively. Phylodynamic analysis revealed an exponential increase in the effective number of infections and Re in both Italy between the 1950s and 1980s, and Albania between the 1990 s and the early 2000s. It seems very likely that HCV-2c reached Italy from Africa at the time of the second Italian colonisation (1936-1941), but did not reach Albania until the period of dramatic migration to Italy in the 1990s.
\end{abstract}

Keywords: epidemiological history of HCV-2; HCV-2 subtypes; evolutionary demography of HCV-2; phylodynamics of HCV-2 in Italy and Albania; HCV-2 Re estimation. 


\section{Introduction}

Hepatitis $\mathrm{C}$ virus (HCV) is one of the main causes of viral hepatitis, which first presents as an acute infection but evolves into chronic disease in $50-70 \%$ of cases [1]. The World Health Organisation (WHO) has estimated that, in 2015, there were 71 million people living with chronic HCV infection at risk of developing cirrhosis and hepatocellular carcinoma (WHO data available at http://www.who.int/hepatitis/publications/global-hepatitis-report2017/en/).

HCV belongs to the positive-sense RNA virus family Flaviviridae, and is characterised by a high degree of genetic variability because it has a high replication rate and is under selective pressure from the immune system, and the error-prone viral protein NS5B, an RNA-dependent RNA-polymerase, lacks proof-reading activity [2]. This has led to the existence of at least 8 main genotypes, more than 80 subtypes and some recombinant form, as stated by the International Committee for the Classification of Viruses (ICTV) in May 2019 (https://talk.ictvonline.org/ictv_wikis/flaviviridae/w/sg_flavi/634/table-1-confirmed-hcv-genotypessubtypes-may-2019) [3].

The geographical distribution of the genotypes and subtypes reflects the diversity of the transmission rates and routes in different areas: epidemic strains such as $1 \mathrm{a}, 1 \mathrm{~b}, 2 \mathrm{a}, 2 \mathrm{~b}, 2 \mathrm{c}$ and $3 a$ spread throughout the world as a result of the use of blood products, invasive procedures and syringe sharing by intravenous drug users, and account for the majority of infections in western countries, whereas endemic subtypes have low transmission rates and generally unknown modes of transmission, thus leading to the local restriction and diversification typical of sub-Saharan Africa and south-east Asia [4-6].

Genotype 2 has been responsible for about $10 \%$ of global infections and has the most variable circulation profile in Europe: HCV-2a and $-2 b$ are the most prevalent subtypes in all of the major European countries (such as Germany, Great Britain and Sweden); subtype 2c is prevalent in Italy and Estonia; in addition to subtypes $2 a, 2 b, 2 c$, new and mixed subtypes have been isolated in The Netherlands, France and Belgium [7].

This diversified distribution makes it fascinating to investigate the molecular evolution of HCV-2 [8,9] because, although the evolutionary and social history of the "endemic" genotype has been satisfactorily reconstructed [10], little is known about the recent spread of the "epidemic" subtypes, particularly HCV-2c. Furthermore, there are some European countries (such as Albania) for which there are hardly any subtype distribution data, although the few studies that have been carried out $[11,12]$ indicate the significant circulation of HCV-2 in particular of subtype 2c.

Direct-acting antivirals (DAAs) have recently become the new standard-of-care for the treatment of chronic HCV infections, and their $>90 \%$ efficacy has induced the WHO to launch a programme aimed at eliminating HCV as a public health threat by 2030 (WHO data available at http://www.who.int/hepatitis/publications/global-hepatitis-report2017/en/). The treatment regimen and the duration administration depend on the viral genotype and subtype, and so the global implementation of DAAs strategies requires a knowledge of the main HCV genotypes and subtypes circulating in different geographical areas. Given the particular nature of the Italian epidemiological picture (in which $-2 \mathrm{c}$ is the most prevalent HCV-2 subtype) and the scarcity of data concerning the circulation of HCV-2 in Albania, the aim of this study was to characterise 245 new $\mathrm{HCV}-2$ isolates in order to reconstruct the evolutionary history of HCV-2 in these two countries phylodynamically and phylogeographically.

\section{Results}

\subsection{Phylogenetic analysis of the global HCV-2 dataset}

The 245 newly characterised HCV NS5B sequences of 208 Italian and 37 Albanian isolates were aligned with the reference sequences as described in Materials and Methods, and analysed using a Bayesian approach. There were a number of significant clades ( $\mathrm{pp}>0.7$ ) corresponding to the known HCV-2 subtypes, but all of the Italian and Albanian strains were included in the three major clades corresponding to the "epidemic" subtypes 2a, 2b and 2c (Figure 1). 


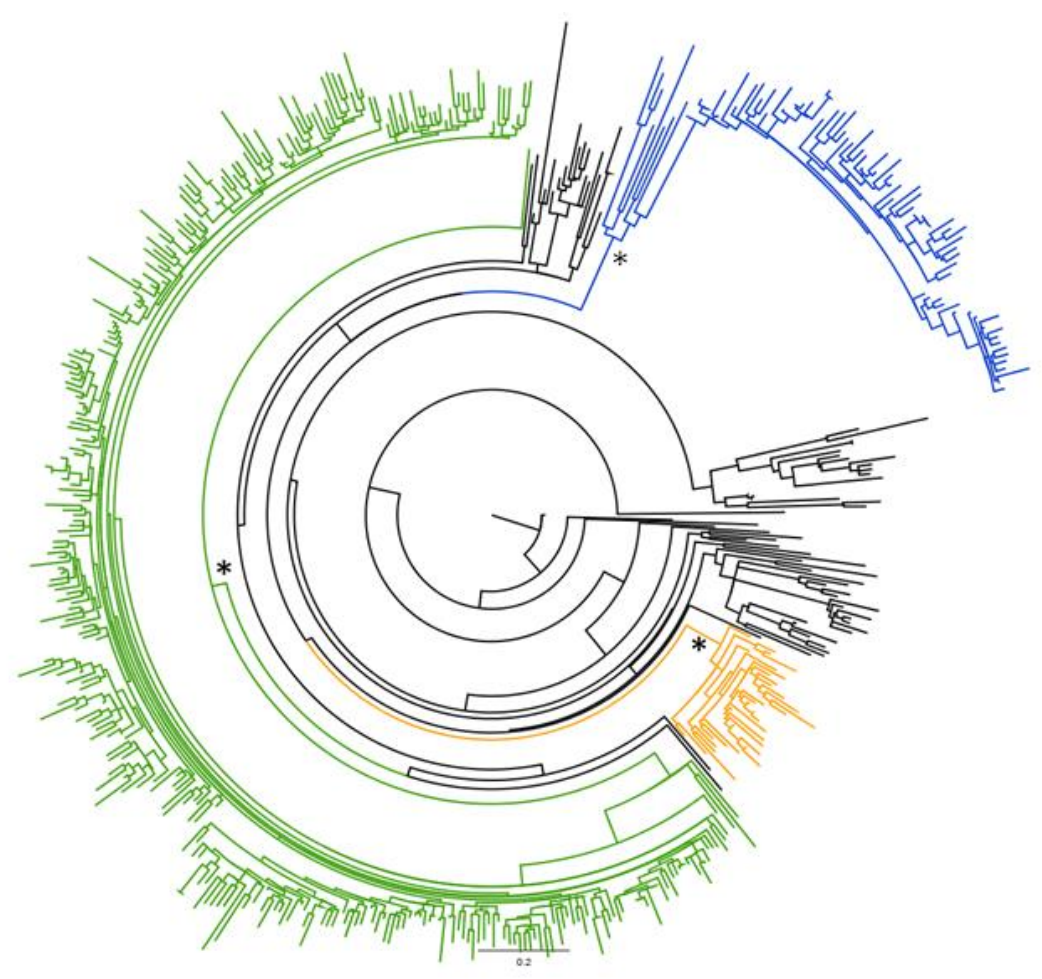

Figure 1. Bayesian phylogenetic tree of the 245 patient sequences and the 273 reference HCV-2 subtype sequences. The different HCV subtypes are highlighted in different colours (2a in yellow; $2 b$ in blue; $2 c$ in green: all of the endemic subtypes in black).*: Significant clades at ML and/or Bayesian analysis corresponding to epidemic subtypes.

The most prevalent subtype was HCV-2c in both Italy (201/208 isolates, 96.6\%) and Albania (36/37 isolates, $97.3 \%)$. The other Italian isolates were classified as $2 \mathrm{~b}(6 / 208,2.9 \%)$ and $2 \mathrm{a}(1 / 208 ; 0.5 \%)$, and the single Albanian isolate outside the 2c clade, was $2 a(1 / 37,2.7 \%)$.

Table 1 shows the main demographic and behavioural risk characteristics of the Italian and Albanian patients infected with HCV-2.

Table 1. Demographic characteristics and reported risks of the study subjects with HCV-2 infection.

\begin{tabular}{|l|l|l|l|}
\hline Characteristics & Italian patients (208) & Albanian patients (37) & Significant (p) \\
\hline Mean age (SD)-Years & 72 years (15.2) & $50.5(14.3)$ & $<0.05$ \\
\hline Males proportion: absolute number (\%) & $81(38.9)$ & $20(54.1)$ & - \\
\hline Females proportion :absolute number (\%) & $127(61.1)$ & $17(45.9)$ & 0,09 \\
\hline Not-known risk-absolute number (\%) & $158(75.9)$ & $31(83.7)$ & 0,3 \\
\hline latrogenic risk-absolute number (\%) & $34(16.3)$ & $6(16.2)$ & - \\
\hline Other risks -absolute number (\%) & $16(7.6)$ & - & - \\
\hline
\end{tabular}

More than $66 \%$ of the study population as a whole (163/245) were aged $>60$ years, but the mean age of the Italian patients was significantly higher than that of the Albanian patients ( $72 \pm 15.2$ vs $50.5 \pm 14.3$ years; $\mathrm{p}<0.005)$. There were more females than males among the Italian patients $(61.1 \%$ vs $38.9 \%)$ but this difference was not significant. Given that in the greater proportion of cases the risk of exposure was not 
known, in the remaining cases the iatrogenic exposure was the most frequent in both Italian and Albanian patients (Table 1).

\subsection{Likelihood mapping of the main dataset}

The main dataset of 419 NS5B sequences used for the phylodynamic and phylogeographical studies was analysed for phylogenetic signals using likelihood mapping (Tree-Puzzle 5.3.rc16). The percentage of dots falling in the central area of the triangle was $8.2 \%$, thus indicating a fully resolved phylogenetic signal (Supplementary Figure 1S).

\subsection{Phylogeographical and phylodynamic analyses}

\subsubsection{Estimated substitution rates and tMRCA}

The mean evolutionary rate of the dataset of HCV-2 NS5B sequences was estimated to be 2.4x10-3 subs/site/year (95\%HPD 1.7-3.1x10-3) using the GTR+I+G substitution model, under constant population size coalescent and strict clock models selected as described above (see Materials and Methods).

Figure 2 shows the dated tree including the patient and reference sequences of genotype 2 using the substitution rate estimated for the whole dataset.

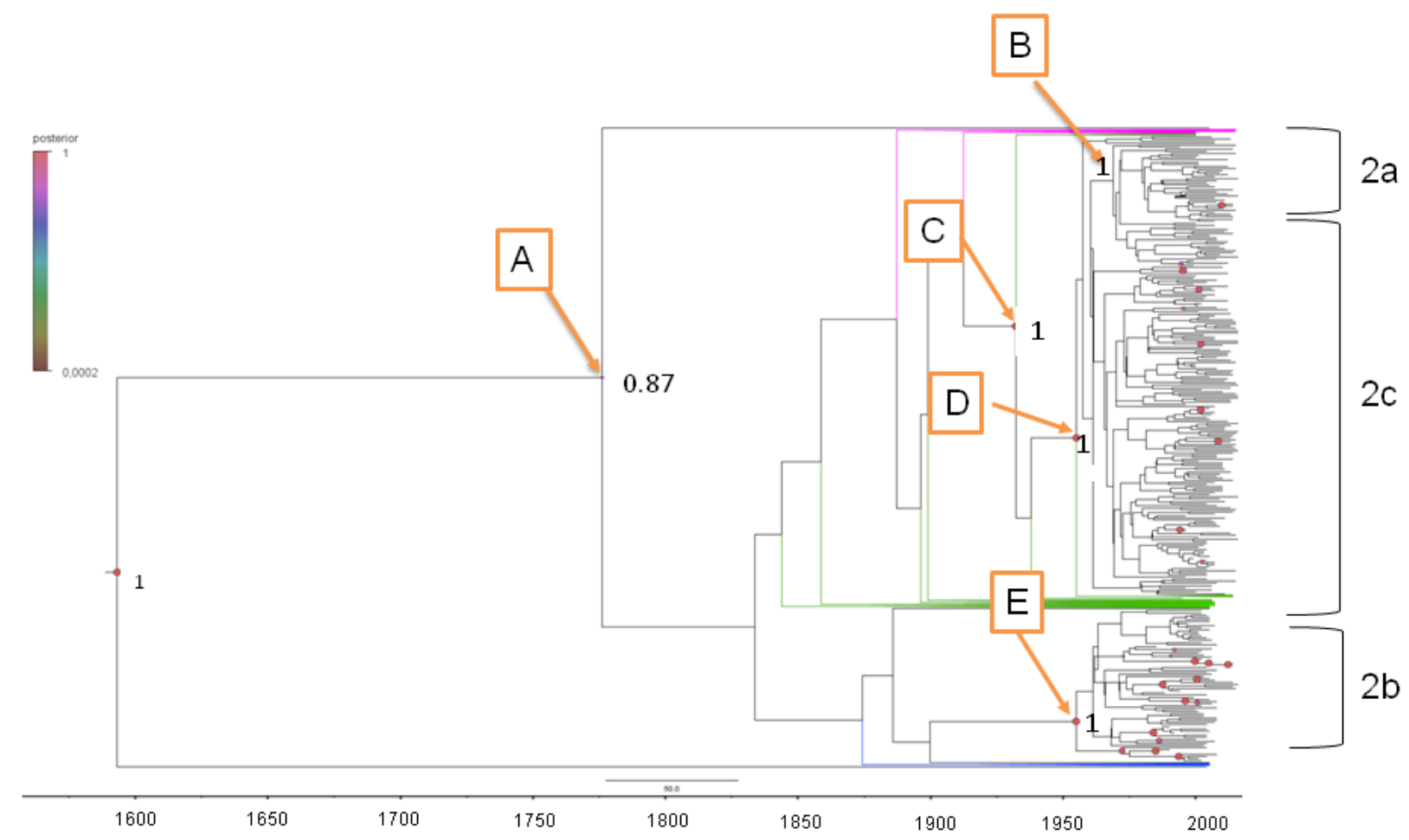

Figure 2. The maximum clade credibility (MCC) tree of the HCV-2 NS5B gene sequences. The letters and arrows indicate the significant internal nodes (posterior probability $\geq 0.8$ ). The scale at the bottom of the tree represents calendar years. The clades corresponding to the three epidemic subtypes $(2 \mathrm{a}, 2 \mathrm{~b}$ and $2 \mathrm{c})$ are highlighted.

Only five nodes in the internal backbone of the tree had posterior probabilities of $>0.7$ (A-E in Figure 2): nodes B, D and E corresponded to the MRCAs of the main "epidemic" HCV subtypes (2a, $2 \mathrm{c}$ and $2 \mathrm{~b}$ respectively), whereas nodes $A$ and $C$ were in a deeper position and respectively represented a common ancestor of all of the HCV-2 strains, and a deeper ancestor of the HCV-2c and HCV-2a clades. Table 2 shows the estimated tMRCAs of the significant internal nodes. 
Table 2. Estimated times and credibility intervals (95\%HPD) of the most recent common ancestors (tMRCAs) and most probable locations, with the state posterior probabilities (spp) of the main clades in the tree shown in Figure 3.

\begin{tabular}{|l|l|l|l|l|l|l|l|l|l|}
\hline Node & Clade & tMRCA & Lower tMRCA & Upper tMRCA & Year mean & Lower year & Upper year & Location & Location Probability \\
\hline Root & & 434,7 & 223 & 684 & 1574 & 1332 & 1793 & Gwinea & 0,49 \\
\hline A & & 227,2 & 137 & 639 & 1781 & 1377 & 1879 & Ghana & 0,89 \\
\hline B & HCV-2b & 61,03 & 49 & 75 & 1948 & 1941 & 1967 & Netherla & 0,99 \\
\hline C & & 83,86 & 61,2 & 109,2 & 1925 & 1906,8 & 1954,8 & Ghana & 0,99 \\
\hline D & HCV-2c & 60,9 & 44,2 & 79,7 & 1948 & 1936,3 & 1971,8 & Italy & 0,83 \\
\hline E & HCV-2a & 56,2 & 39,4 & 73,1 & 1954 & 1942,9 & 1976,6 & Indonesi & 0,38 \\
\hline
\end{tabular}

The tree-root tMRCA was estimated to be a mean 435 years ago (YA: 95\%HPD 223-684 YA), corresponding to the year 1574 (CI 1332-1793). The tMRCA estimates of the three main clades corresponding to nodes B, D and E were between 1948 (2b and 2c) and 1954 (2a). The estimated tMRCA of the highly significant node $\mathrm{C}$ preceding the common ancestor of $\mathrm{HCV}-2 \mathrm{C}$ was an average of $83.9 \mathrm{YA}$, corresponding to the year 1925 (95\%HPD 1907-1954).

\subsubsection{Phylogeography of HCV-2}

Figure 3 shows the phylogeographical Baysian tree indicating the most probable locations of the internal nodes by different branches colours.

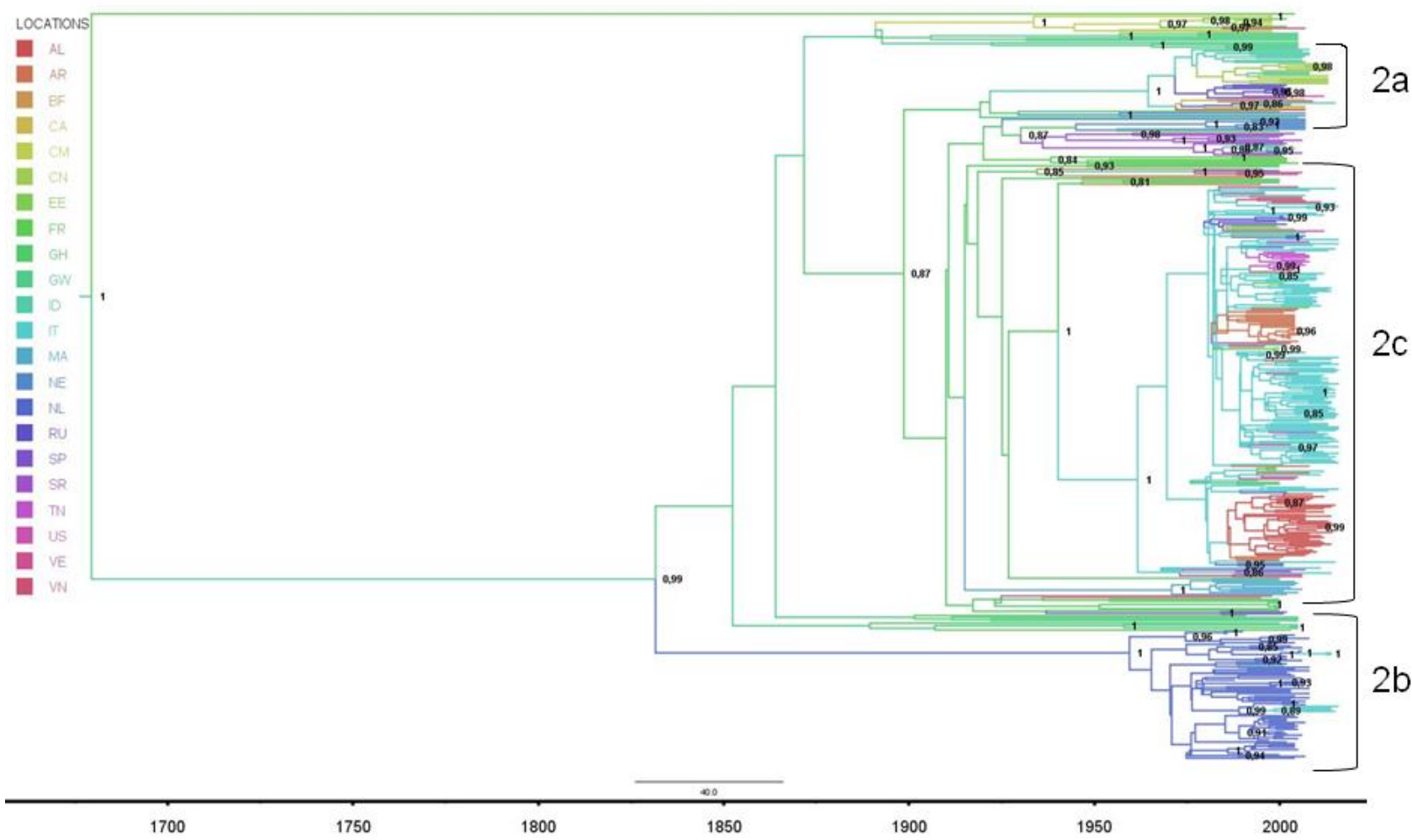

Figure 3. The Bayesian phylogeographical MCC tree of $419 \mathrm{HCV}-2$ NS5B gene sequences. The branches are coloured on the basis of the most probable location of the descendent nodes (see colour code in upper left inset). The initials on the internal nodes correspond to the country code of the most probable location, and the 
shapes of the internal nodes have been sized in proportion with their posterior probabilities. The scale at the bottom of the tree represents calendar years.

The most probable location of the tree root was Guinea Bissau (st $p p=0.49$ vs France st $p p=0.32)($ Table 2). The most probable locations of the MRCAs of the epidemic subtypes were Italy (st pp=0.83) for HCV-2c, and The Netherlands (st $p p=0.99$ ) for $\mathrm{HCV}-2 \mathrm{~b}$; Indonesia and The Netherlands had similar posterior probabilities of being the location of the HCV-2a ancestor (Indonesia st pp=0.38; The Netherlands st $\mathrm{pp}=0.24)$. The second highly significant internal node $(\mathrm{C})$ of $\mathrm{HCV}-2 \mathrm{c}$ had Ghana as the most probable location (st $\mathrm{pp}=0.99$ ). The Albanian isolates tended to group into two sub-clades supported by low posterior probabilities ( $\mathrm{pp}>0.50)$.

Table 3 shows the 33 identified significant non-zero rates (BF $>3$ ), most of which included Italy (10), Africa (8), France (5), and The Netherlands (4).

Table 3. Significant non-zero rates of different sampling locations supported by a BF of $>3$.

\begin{tabular}{|c|c|c|c|}
\hline Rates & Location 1 & Location 2 & Bayes Factor \\
\hline 1 & IT & VE & 86827.07 \\
\hline 2 & IT & $\mathrm{NL}$ & 86827.07 \\
\hline 3 & ID & SR & 86827.07 \\
\hline 4 & $A R$ & IT & 86827.07 \\
\hline 5 & IT & $\mathrm{TN}$ & 14463.14 \\
\hline 6 & $\mathrm{CN}$ & ID & 7226.74 \\
\hline 7 & ID & $\mathrm{NL}$ & 5417.65 \\
\hline 8 & $\mathrm{AL}$ & IT & 4560.70 \\
\hline 9 & FR & IT & 3765.86 \\
\hline 10 & $\mathrm{CM}$ & FR & 2108.32 \\
\hline 11 & $\mathrm{BF}$ & $\mathrm{GH}$ & 161.63 \\
\hline 12 & FR & GW & 96.64 \\
\hline 13 & $\mathrm{RU}$ & US & 73.69 \\
\hline 14 & $\mathrm{GH}$ & $\mathrm{NE}$ & 47.67 \\
\hline 15 & $\mathrm{GH}$ & IT & 29.40 \\
\hline 16 & $\mathrm{GH}$ & SP & 22.94 \\
\hline 17 & $\mathrm{GH}$ & SR & 22.35 \\
\hline 18 & $\mathrm{GH}$ & $\mathrm{MA}$ & 21.54 \\
\hline 19 & FR & $\mathrm{VN}$ & 13.71 \\
\hline 20 & $\mathrm{EE}$ & $R U$ & 13.01 \\
\hline 21 & FR & $\mathrm{GH}$ & 12.21 \\
\hline 22 & $\mathrm{GH}$ & GW & 11.74 \\
\hline 23 & IT & $\mathrm{RU}$ & 9.94 \\
\hline 24 & ID & $R U$ & 7.14 \\
\hline 25 & $\mathrm{AL}$ & $C A$ & 6.48 \\
\hline 26 & $\mathrm{NL}$ & $\mathrm{RU}$ & 6.38 \\
\hline 27 & CA & $\mathrm{NL}$ & 5.77 \\
\hline 28 & $\mathrm{EE}$ & IT & 5.65 \\
\hline 29 & CM & $\mathrm{VN}$ & 5.22 \\
\hline 30 & CA & ID & 4.25 \\
\hline 31 & NE & SR & 4.15 \\
\hline 32 & CA & IT & 3.68 \\
\hline 33 & ID & $\mathrm{VN}$ & 3.43 \\
\hline
\end{tabular}


In particular, Italy showed highly significant linkages with Albania $(\mathrm{BF}=4561)$, Argentina $(\mathrm{BF}=86827)$, The Netherlands $(\mathrm{BF}=86827)$, Venezuela $(\mathrm{BF}=86827)$, Tunisia $(\mathrm{BF}=14463)$, France $(\mathrm{BF}=3766)$, and Ghana $(\mathrm{BF}=29.5)$ (highlighted in Table 3).

The phylogeographical reconstruction of migration flows (a - d Figure 4) showed that, after its origin in western Africa (Guinea Bissau) in the XVI century, the HCV-2 genotype migrated to central Africa, (in particular Ghana for HCV-2a and -2c). From Africa, it moved to Europe and had reached France, The Netherlands and Italy by the 1930s-1950s.
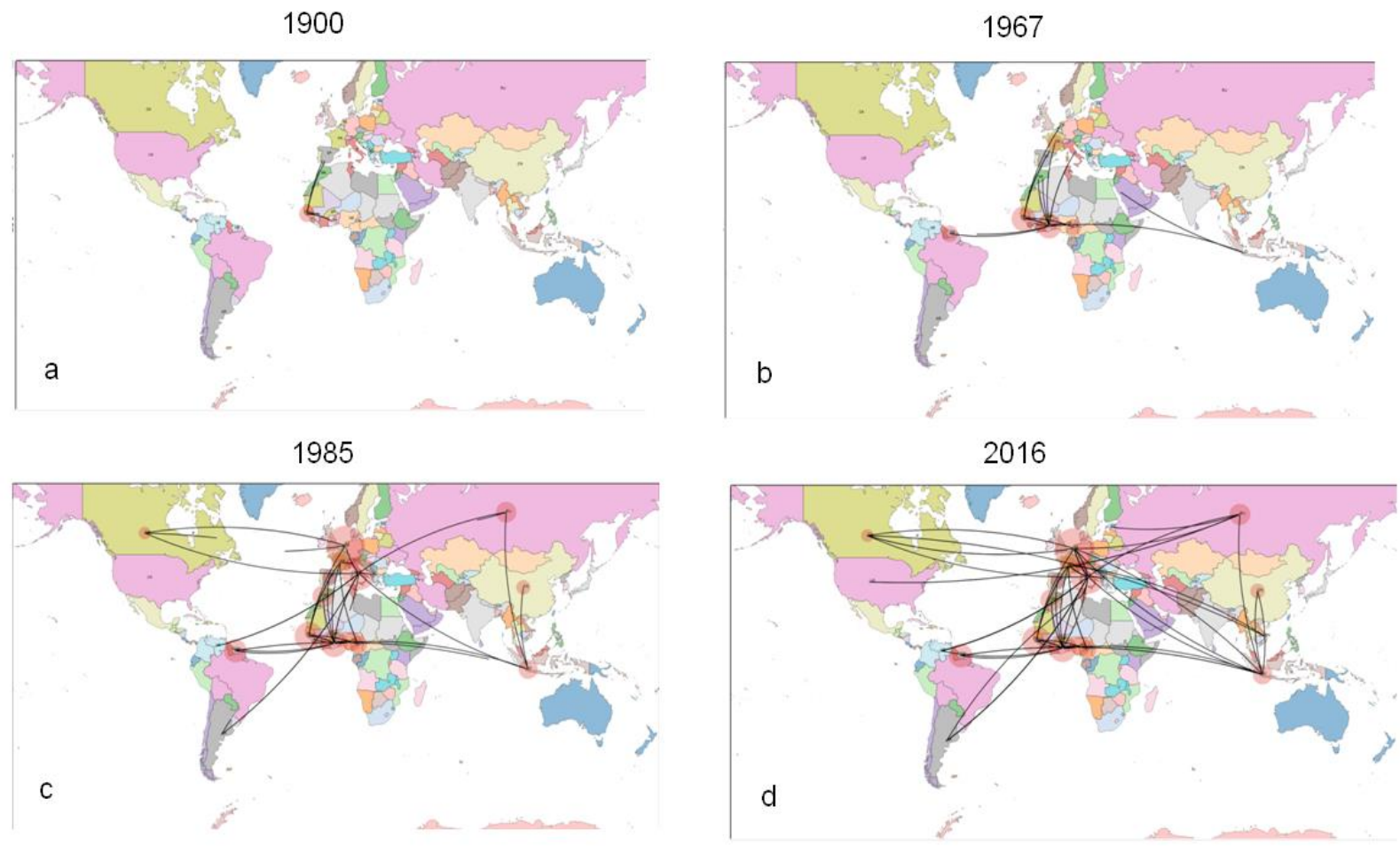

Figure 4. Significant non-zero migration rates of HCV-2 worldwide. Only the rates supported by a BF $>3$ are shown. The map was reconstructed using SPREAD (see Materials and Methods). The four panel (a-d) correspond to snapshots of the four different times indicated in correspondence with the panel.

\subsubsection{Evolutionary demography and R0/Re estimates in Italy and Albania}

The Italian and Albanian HCV-2c isolates were separated for further independent phylodynamic analyses. The skyline plot of the Italian sequences showed a growth in the effective number of infections during the period from the 1940s to the mid-1960s, followed by a plateau (Figure 5a). The direct estimate of HCV Re using the birth-death model and five intervals of time showed that it started to grow in the 1940s, reached a peak of 3.2 (95\%HPD 1.1-7.5) in the period between the 1980s and early 2000s, after which it levelled off before starting to decrease only recently (Figure $5 \mathrm{~b}$ ). The model estimate indicated that the origin of the HCV genotype 2 epidemic in Italy was 70.5 YA (42.7-100.0 YA). The estimate of the becoming-noninfectious rate was 0.199 ( $\mathrm{CI}=0.02-0.47)$, corresponding to a mean time of infectiousness of five years (CI 2-50 years).

The skyline plot analysis of the Albanian sequences (Figure 5c) showed an exponential growth in the effective number of infections between the early 1990s and the mid-2000s, after which it reached a plateau. The birth-death model estimate of the trend of Re was similar: growth between the 1990s and early 2000s, followed by a rapid decrease. The origin of the epidemic was estimated to be 40.6 YA (26.3-86.9), corresponding to 1975 (Figure 5d). 
a

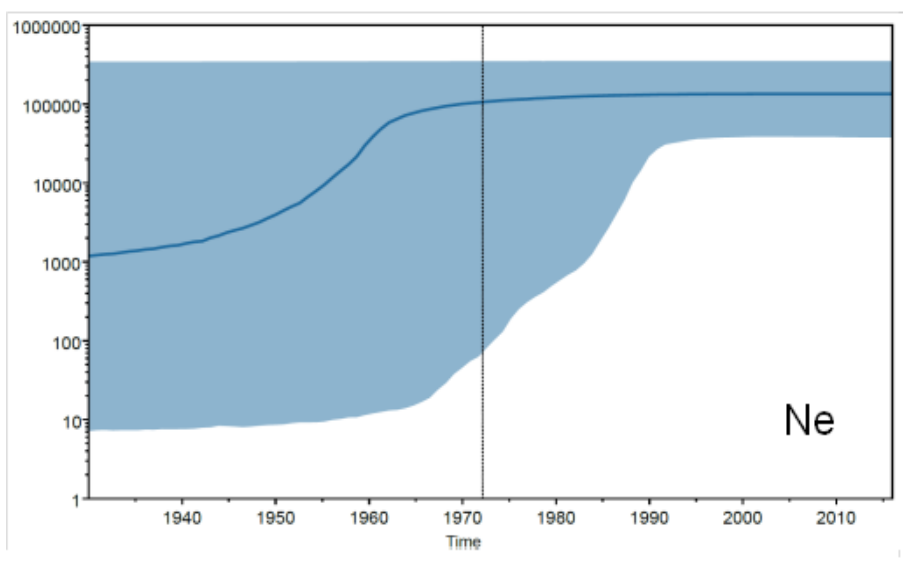

b

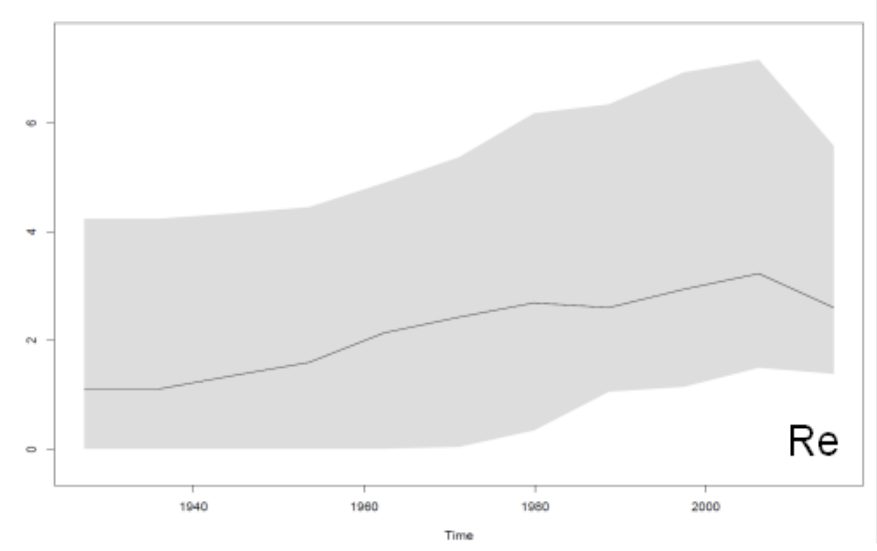

C

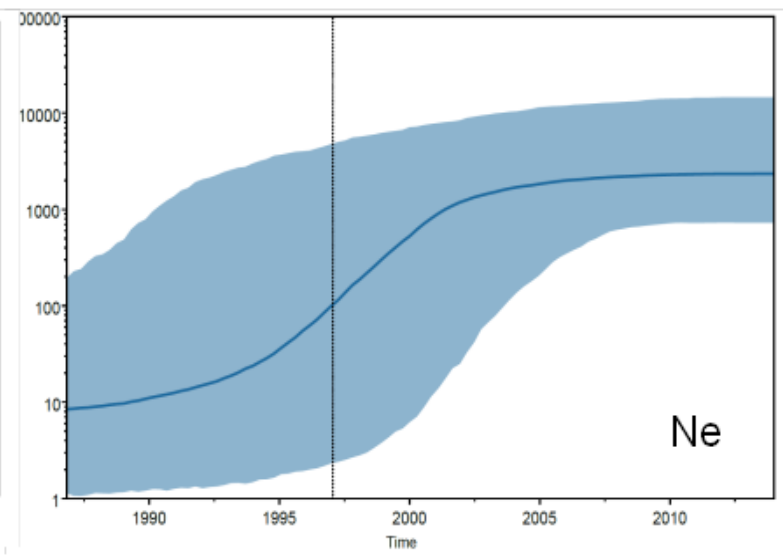

d

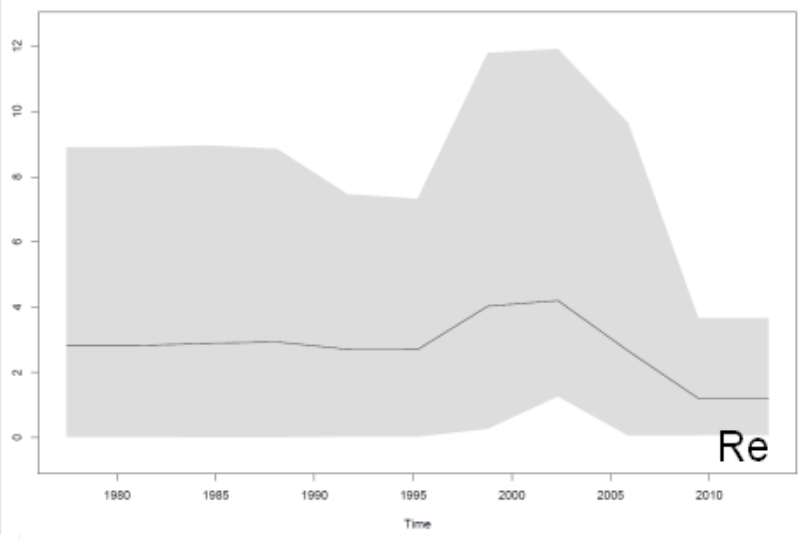

Figure 5. HCV-2 in Italy (a and b) and Albania (c and d). Bayesian skyline plots (a and c) and birth-death skyline plots ( $b$ and $d$ ) for the estimated median values and HPD intervals of effective population size (Ne) and effective reproduction numbers (Re).

\section{Discussion}

The aim of this study was to reconstruct the epidemiological history of HCV-2 and its "epidemic" subtypes in Europe, particularly HCV-2c as it is the most frequent HCV-2 subtype in Italy and Albania. To do this, we newly characterised NS5B sequences from 208 Italian and 37 Albanian isolates obtained from patients with chronic HCV-2 infection over a period of 25 years. A subset of these sequences was subsequently aligned with reference sequences obtained in different parts of the world at different times and retrieved from public databases in order to allow phylodynamic and phylogeographical analyses.

The vast majority (97\%) of the isolates obtained from Italian patients were $\mathrm{HCV}-2 \mathrm{c}$; the only exceptions were six isolates forming a significant monophyletic group of subtype $2 b$ dating back to 1989, with an outgroup of six Dutch sequences obtained between the end of the 1980s and the early 2000s. This is in line with the findings of other studies showing that subtype $2 \mathrm{c}$ is very frequently found in Italy, whereas it represents only a minority of the isolates collected in other European countries [7]. Interestingly, the 2c strain also accounted for $97 \%$ of the isolates taken from HCV-2 positive Albanian patients. There are only a few studies of the prevalence of HCV genotypes in Albania, but all of them have found that HCV-2c is the most frequent subtype after $\mathrm{HCV}-1 \mathrm{~b}$, infecting $9-18 \%$ of all of the Albanian patients with chronic HCV infection $[11,13,14]$.

Another main finding is that the Italian patients infected by HCV-2 were older than their Albanian counterparts: $66 \%$ were more than 60 years old. Other studies have found that HCV-2 is highly prevalent among elderly Italians [15-17]; it was also highly prevalent among Italian children, but its prevalence has been decreasing in those born after 1990 [18]. A number of epidemiological studies suggested that HCV-2 used to be even more prevalent in Italy in the past [15-17,19-23], which would explain its widespread 
prevalence among elderly Italians, but it has now been overtaken by other genotypes in younger subjects, who have mainly been infected as a result of intravenous drug use or contaminated blood transfusions [24].

The penetration of HCV-2 in Albania is probably more recent (see below), which would explain its greater frequency among younger Albanians with HCV-2 infection.

These data are in line with the rapidly changing epidemiology of HCV genotypes in Europe [25].

Several authors have tried to reconstruct the origin and global spread of genotype 2 using various phylogenetic approaches. One of the most important studies is that of Markov et al. [26], who suggested that HCV-2 originated in western Africa (Guinea Bissau) between 1400 and 1500 AD, and then migrated eastward to central Africa. In a subsequent study, the same authors demonstrated the central role of European (particularly Dutch and French) colonialism in the spread of endemic HCV-2 subtypes throughout the New World, whereas the dissemination of the epidemic subtypes $2 a, 2 b$ and $2 c$ did not occur until the XX century, and was due to the use of contaminated blood products or intravenous drug use [10], as shown by their clustering on the basis of the mode of transmission. Other authors have confirmed the western African origin of HCV-2 and underlined the role of colonialism in its spread $[8,26,27]$.

These studies were mainly aimed at reconstructing the history of endemic subtypes, but they were less effective in assessing the history of epidemic subtypes, possibly because of differences in the time scales of the evolution of the two groups of subtypes. It has been shown that the time dependency of substitution rates leads to overestimates when timescales are short (as in the case of the epidemic strains of $\mathrm{HCV}$ ) and underestimates when timescales are long (as in the case of the endemic strains) [28]; however, most of the studies cited above used the same NS5B fragment substitution rate $\left(5 \times 10^{-4}\right.$ subs/site/year) for both endemic and epidemic subtypes. This substitution rate was independently estimated using HCV-1 isolates collected after an epidemic event due to accidental transmission caused by a single batch of anti-rhesus immunoglobulin in Ireland in 1978 [29], and it was used by Pybus et al. in their first reconstruction of the phylodynamics of HCV genotypes [30]. However, various factors can affect the substitution rate of viruses [31], including the epidemiological/ecological dynamics of the infection in the host population [32-34] and the main routes of transmission [35], two characteristics that clearly distinguish endemic and epidemic HCV geno/subtypes [4].

As the aim of this study was to reconstruct the molecular evolution of epidemic HCV-2 subtypes by including a large number of Italian and Albanian HCV-2c sequences newly characterised by us, we estimated the substitution rate on the basis of sampling years, and obtained a mean value of $2.4 \times 10^{-3}$ subs/site/year (CI 1.7-3.1 $\times 10^{-3}$ subs/site/year). This is faster than the mean rate used by other authors, but in line with a recent review of HCV evolution that suggested a mean evolutionary rate of about $1 \times 10^{-3}$ subs/site/year [36]. It also confirms the observation that different genotypes/subtypes may have different substitution rates as HCV-2 has evolved much more rapidly than the other genotypes [37].

Despite our use of a faster rate, our phylogeographical reconstruction confirmed that the most probable origin of HCV-2 was western Africa (Guinea Bissau) at least 500 years ago, after which it spread eastward through central Africa to Ghana, an important centre of its further spread out of Africa [26,27]. Our estimates of the tMRCAs of the significant clades and analyses of the significant linkages between locations suggest that the epidemic subtypes migrated to Europe between the 1930s and 1950s, when HCV-2 reached The Netherlands, Italy and France, which became the main centres of the further worldwide spread of the epidemic up to the 1980s.

Our phylogeographical analysis indicates that HCV-2c has two internal node MRCAs: one dating back to the 1950s with a more probable location in Italy, and another (including also HCV-2a subtype) dating back to the 1930s with Africa (i.e. Ghana) as the most likely location. Interestingly, other authors have suggested Ghana as a further possible location of the origin of HCV-2 [27], thus underlining the central role of the country in its dissemination. The analysis of genetic flow rates suggests that HCV-2c moved to Italy between the 1930s (when its ancestor existed in Africa) and the 1950s (when the common ancestor of the currently circulating HCV-2c appeared in Italy), after a largely unknown journey lasting at least 20 years.

The main obstacle to reconstructing the route taken by this original strain before its arrival in Italy is the lack of isolates from other African countries. Nevertheless, as in the case of the other HCV-2 subtypes, it is reasonable to assume that European colonial history played an important role in the arrival of $\mathrm{HCV}-2 \mathrm{c}$ in Italy [38], even if the history of Italy in Africa is more restricted in time and space than that of other western 
countries: from the 1890s to the end of World War II in regions of eastern Africa (Somalia, Eritrea, Ethiopia) and northern Africa (Libya). One recent study has shown a high prevalence of HCV-2c in Ethiopia [39], where Italian soldiers and often resident migrant workers were present from 1936 to 1941. It is therefore possible that, after the end of World War II, the Italians who returned to Italy carried HCV-2c infection with them. Further studies including more African isolates would be useful to clarify how HCV-2c reached Ethiopia from Ghana, and the role played by eastern Africa in allowing HCV-2c to reach Italy.

The Bayesian skyline plot of population dynamics showed that, like that of other HCV subtypes in other western countries [10,40], the exponential growth of HCV-2c infection in Italy started in the 1950s and continued until the 1980s. We also traced the changes in the Re of epidemic HCV-2c in Italy using a birthdeath model to estimate the effective replication number and other important epidemiological parameters. The results of these analyses indicate that, over a period of about 70 years (the estimated origin of the epidemics), Re rapidly increased between the 1950s and the 1980s, continued to grow at a slower rate until the 2000s, and then started to decline.

On the contrary, the skyline analysis showed that exponential growth of both the effective number of infections and the Re in Albania started later, between the early 1990s and the 2000s- Re began to decrease in 2000s. Albania was associated with Italian colonisation during the twenty years of the fascist period, but our estimates of the tMRCAs of the significant Albanian clades suggests that HCV-2c reached Albania from Italy more recently, between the 1990s and 2010. During that period, Albania was going through a political and economic crisis due to the fall of the communist regime, and hundreds of thousands of Albanian migrated to Italy [41], particularly in the early 1990s and the late 1990s/early 2000s. This suggests that the infection was exported from Italy to Albania when infected Albanian migrants returned to their native country.

In conclusion, the findings of this study (the first to report the trend of the effective reproductive number of HCV-2 in two European countries) contribute to reconstructing the history of the spread of epidemic HCV-2 subtypes to Europe in the XX century, and show that Italy was an important centre of the spread of HCV-2c in the Mediterranean area.

\section{Material and Methods}

\subsection{Ethics statement}

This retrospective research was conducted on serum samples collected for clinical purposes and stored in the participating centers. All data used in the study were previously anonymized, according to the requirements set by Italian Data Protection Code (leg. Decree 196/2003) and by the General authorizations issued by the Data Protection Authority.

Approval by Ethics Committee was deemed unnecessary because, under Italian law, such an approval is required only in the hypothesis of prospective clinical trials on medical products for clinical use (art. 6 and art. 9, leg. Decree 211/2003). Written informed consent for medical procedures/interventions performed for routine treatment purposes was collected for each patient.

\subsection{HCV-2 positive patients and sequences}

The 245 HCV-2 NS5B sequences newly characterised in our laboratory were obtained from 208 Italian samples taken from patients attending the Infectious Diseases Department of L. Sacco University Hospital in Milan or the Virology Unit of the Campus Bio-Medico University in Rome, and 37 samples taken from Albanian patients attending the National Blood Transfusion Centre of Tirana. The data concerning the samples and patients (age, country of birth and town of residence) were collected between 2001 and 2016. Patients plasma samples with $>1000$ IU of $\mathrm{HCV}$ RNA/mL were genotypically analysed using a VERSANT® HCV Genotype 2.0 - LiPA kit (Siemens Healthcare, Erlangen, Germany), and the same samples were further characterised by means of NS5B gene sequencing. 


\subsection{Sample processing and HCV RNA sequencing}

Viral RNA was extracted from the patients' serum $\left(200 \mu \mathrm{L}\right.$ stored at $\left.-80^{\circ} \mathrm{C}\right)$ using NucleoMag 96 Virus (Macherey-Nagel, Düren, Germany) and automated KingFisher ${ }^{\mathrm{TM}}$ Magnetic Particle Processors (Thermo Fisher Scientific Inc., Waltham, MA, USA) in accordance with the manufacturer's instructions. Serum samples from healthy subjects were used as negative controls. The RNA was eluted in $50 \mu \mathrm{L}$ of nucleasefree distilled water, and reverse transcribed using the SuperScript III reverse transcriptase protocol (Thermo Fisher Scientific): the cDNA was amplified by means of nested polymerase chain reaction (PCR) using GoTaq ${ }^{\circledR}$ DNA Polymerase (Promega, Madison, WI). The primers for the first and second rounds of NS5B amplification and the PCR conditions have been previously described [42,43].

The fragments obtained by means of PCR were purified using a commercial purification kit (QIAquick PCR Purification Kit, Qiagen, Hilden, Germany), and then sequenced bi-directionally using a BigDye Terminator Kit version 3.1 (Applied Biosystems, CA, USA) in accordance with the manufacturer's instructions.

The sequencing products were purified from a $10 \mu \mathrm{L}$ sample by means of precipitation in an ethanol/sodium acetate mixture. Finally, the sequences were determined using an automated DNA sequencer (ABI PRISM 3130 XL Genetic Analyser, Applied Biosystems).

\subsection{HCV-2 NS5B datasets}

The 245 newly characterised NS5B sequences were aligned for phylogenetic analysis with 273 reference sequences (representative of previously described HCV-2 subtypes $2 \mathrm{a}, 2 \mathrm{~b}, 2 \mathrm{c}, 2 \mathrm{e}, 2 \mathrm{f}, 2 \mathrm{i}, 2 \mathrm{j}, 2 \mathrm{~m}$ and $2 q$ ) retrieved from public databases (http://www.ncbi.nlm.nih.gov/genbank/).

Bayesian phylogeographical and phylodynamic analyses were carried out using a dataset of 419 sequences, including a total of 112 Italian NS5B sequences randomly selected from the main dataset and all of the Albanian sequences, which were aligned with 270 reference sequences. The reduction in the number of Italian sequences was necessary in order to limit the effects of sampling errors due to the number of taxa per location on the assessment of the posterior probability of the root location. The reference viral sequences were selected on the basis of the following inclusion criteria: 1) they had been published in peer-reviewed journals; 2) there was no uncertainty about the sub-genotype assignment of each sequence and all were classified as non-recombinant; and 3) the city/state of origin were known and clearly stated in the original publication. The accession numbers, sampling localities and characteristics of the isolates included in the dataset are summarised in Supplementary Table S1. The sampling dates ranged from 1985 to 2016.

Two other datasets were constructed: a subset including 199 Italian HCV-2c NS5B partial sequences, and a subset including $36 \mathrm{HCV}-2 \mathrm{c}$ NS5B partial sequences collected in Albania.

The sampling locations of the isolates included in the main dataset were Albania (AL, $n=37)$, Argentine $(A R, n=26)$, Burkina Faso (BF, $n=5)$, Canada (CA, n=3), Cameroon $(C M, n=7)$, China $(C N, n=10)$, Estonia (EE, $n=3)$, France (FR, $n=18)$, Ghana ( $\mathrm{GH}, \mathrm{n}=18)$, Guinea Bissau (GW, $\mathrm{n}=16)$, Indonesia (ID, $n=15)$, Italy (IT, $n=112)$, Morocco (MA, n=9), Nigeria (NE, $n=9)$, The Netherlands (NL, $n=76)$, Russia (RU, $n=11)$, Spain (SP, $\mathrm{n}=2)$, Suriname $(\mathrm{SR}, \mathrm{n}=8)$, Tunisia $(\mathrm{TN}, \mathrm{n}=15)$, the United States (US, $\mathrm{n}=2)$, Venezuela $(V E, \mathrm{n}=15)$, and Vietnam $(\mathrm{VN}, \mathrm{n}=2)$.

\subsection{Likelihood mapping analysis}

In order to obtain an overall impression of the phylogenetic signals in the partial NS5B gene sequences, we made a likelihood-mapping analysis [44] of 10,000 random sets of four sequences (quartets) generated using TreePuzzle software [45]. If the frequency of dots within the central area (star-like trees) of the triangle is more than $30 \%$, it is likely that the data includes a high level of noise. 


\subsection{Bayesian phylogenetic and phylogeographical reconstructions}

The main dataset and the subsets were aligned using the ClustalW software included in BioEdit [46], followed by manual editing (final alignment length $=211$ nucleotide).

The JModelTest was used to select the simplest evolutionary model fitting the data, which was the GTR $+\mathrm{I}+\mathrm{G}$ model of nucleotide substitutions for the main dataset and the Albanian subset, and the GTR $+\mathrm{G}$ model for the Italian subset.

The phylogenetic tree, model parameters, evolutionary rates and population growth were coestimated using the Bayesian Markov chain Monte Carlo (MCMC) method implemented in BEAST v.1.8.4 [47]. Statistical support for specific clades was obtained by calculating the posterior probability of each monophyletic clade. Four simple parametric demographic models (constant population size, and exponential, expansion and logistic population growth) and a piecewise-constant Bayesian skyline plot (BSP) under both a strict and a relaxed (uncorrelated log-normal) clock were compared as coalescent priors [47].

The phylogeographical reconstruction was made using the continuous-time Markov Chain (MCC) process over discrete sampling locations implemented in BEAST [48], and the Bayesian Stochastic Search Variable Selection (BSSVS) model that allows diffusion rates to be zero with a positive prior probability. Comparison of the posterior and prior probabilities that the individual rates would be zero provided a formal Bayesian factor (BF) for testing the significance of the linkages between loca tions: rates with a BF of $>3$ were considered well supported and assumed to be the migration pathway.

The HCV-2 dataset and the Italian HCV-2c subset were investigated by running two independent MCMCs for 500 million generations, with sampling every 50,000 generations; the Albanian HCV-2c subset was investigated using 50 million generations, with sampling every 5,000 generations. The data were combined using LogCombiner v. 1.80 in the BEAST package. Convergence was assessed on the basis of the effective sampling size (ESS) after a $10 \%$ burn-in using Tracer v. 1.5 software (http://tree.bio.ed.ac.uk/software/tracer/). Only ESS's of $\geq 200$ were accepted. Uncertainty in the estimates was indicated by $95 \%$ highest posterior density (95\% HPD) intervals, and the best fitting models were selected using the BF and marginal likelihoods implemented in BEAST [49]; in accordance with Kass [50], only $2 \operatorname{lnBF}$ values of $\geq 6$ were considered significant. The trees were summarised in a target tree using the Tree Annotator program included in the BEAST package and selecting the tree with the maximum product of posterior probabilities (maximum clade credibility) after a 10\% burn-in. The estimates of the time of the most recent common ancestor (tMRCA) were expressed as the mean number of years and $95 \%$ HPD before the most recent sampling dates (which corresponded to 2016). The final trees were visualised using FigTree v. 1.4 (available at http://tree.bio.ed.ac.uk/software). In order to visualise diffusion rates over time, it is also possible to convert the location-annotated MCC tree to a GeoJSON data format suitable for viewing with georeferencing software and, using the new SPREAD3 analytical tool, the MCC tree was converted to a JavaScript object notation (JSON) file. The visualisation was rendered using a Data Driven Document (D3) library [51].

\subsection{Birth-death skyline plot estimate of effective reproduction number (Re)}

In order to make a direct estimate of the changes in effective reproductive number (Re) over time, we reconstructed the phylogenetic tree of the Italian and Albanian HCV-2c isolates using a birth-death skyline plot [52], which makes it possible to infer the transmission rate (lambda), the death/recovery rate (delta), and the sampling proportion (p).

We used a serial birth-death skyline plot (a forward-in-time model allowing the parameters to change in a piecewise fashion) and a lognormal prior probability distribution of the reproductive number (R), with a mean value $(\mathrm{M})$ of 0.0 , a variance $(\mathrm{S})$ of 1.25 , and a total of five dimensions. The becoming-noninfectious parameter was estimated using a lognormal prior with $\mathrm{M}=0.5$ and $\mathrm{S}=1.5$, so that the $95 \%$ probability of the infectious period fell between 0.1 and 19.4 years, with a median of five years. The prior of the sampling probability followed a beta distribution with an alpha value of 1 and a beta value of 100, which corresponds to a minority of sampled cases in relation to all cases (from 0.0005 to 0.03 ). 
As there are no data concerning the origin of the outbreak of HCV-2 infection in Italy and it is known that the circulation of HCV-2 has been widespread over the last 100 years, we used a lognormal prior with $\mathrm{M}=4$ and $\mathrm{S}=0.4$. The highest probability was a period between approximately 28.3 and 105 years ago, with a median of about 54.6 years.

Similar values were used for the serial birth-death skyline plot of the Albanian sequences, with the exception that there were four dimensions of Re and the prior for the origin was between 15 and 100 years ago.

Author Contributions: E.E. and G.Z. designed the research; E.E., G.Z., A.M., M.A., S.R., R.S., CDV and P.D. performed the research; E.E., G.Z., A.C., M.C., S.B., and CDV analyzed the data; and E.E., G.Z., M.G., V.M., E.R., and E.T. wrote the manuscript. All authors have read and approved the final version of the manuscript.

Acknowledgments: This study was partially financed by the Italian Ministry for Education, University and Research "NANOMAX" Bandiera project (2013-2015): Grant No. G42I12000180005) to GZ.

Conflicts of Interest: The authors declare no conflict of interest.

\section{Abbreviations}

GT

AL

AR

BF

CA

$\mathrm{CM}$

$\mathrm{CN}$

EE

FR

GH

GW

ID

IT

MA

NE

NL

RU

SP

SR

TN

US

VE

$\mathrm{VN}$

HPD

tMRCA

GTR+I+G

YA
Genotype

Albania

Argentine

Burkina Faso

Canada

Cameroon

China

Estonia

France

Ghana

Guinea Bissau

Indonesia

Italy

Morocco

Nigeria

The Netherlands

Russia

Spain

Suriname

Tunisia

The United States

Venezuela

Vietnam

High Posterior Density

time of the Most Recent Common Ancestor

Generalised time reversible + proportion of invariable sites + gamma distribution Years Ago 


\section{References}

1. Snow, K.K.; Bell, M.C.; Stoddard, A.M.; Curto, T.M.; Wright, E.C.; Dienstag, J.L. Processes to manage analyses and publications in a phase III multicenter randomized clinical trial. Trials 2014, 15, 159.

2. Sesmero, E.; Thorpe, I.F. Using the Hepatitis C Virus RNA-Dependent RNA Polymerase as a Model to Understand Viral Polymerase Structure, Function and Dynamics. Viruses 2015, 7, 3974-3994.

3. Smith, D.B.; Bukh, J.; Kuiken, C.; Muerhoff, A.S.; Rice, C.M.; Stapleton, J.T.; Simmonds, P. Expanded classification of hepatitis $C$ virus into 7 genotypes and 67 subtypes: updated criteria and genotype assignment web resource. Hepatology 2014, 59, 318-327.

4. Pybus, O.G.; Markov, P.V.; Wu, A.; Tatem, A.J. Investigating the endemic transmission of the hepatitis C virus. International journal for parasitology 2007, 37, 839-849.

5. Messina, J.P.; Humphreys, I.; Flaxman, A.; Brown, A.; Cooke, G.S.; Pybus, O.G.; Barnes, E. Global distribution and prevalence of hepatitis C virus genotypes. Hepatology 2015, 61, 77-87.

6. Simmonds, P. Genetic diversity and evolution of hepatitis C virus - 15 years on. Journal of General Virology 2004, 85, 3173-3188, doi:https://doi.org/10.1099/vir.0.80401-0.

7. Welzel, T.M.; Bhardwaj, N.; Hedskog, C.; Chodavarapu, K.; Camus, G.; McNally, J.; Brainard, D.; Miller, M.D.; Mo, H.; Svarovskaia, E., et al. Global epidemiology of HCV subtypes and resistance-associated substitutions evaluated by sequencing-based subtype analyses. Journal of hepatology 2017, 67, 224-236.

8. $\quad$ Cantaloube, J.F.; Gallian, P.; Laperche, S.; Elghouzzi, M.H.; Piquet, Y.; Bouchardeau, F.; Jordier, F.; Biagini, P.; Attoui, H.; de Micco, P. Molecular characterization of genotype 2 and 4 hepatitis $\mathrm{C}$ virus isolates in French blood donors. J Med Virol 2008, 80, 1732-1739.

9. Thomas, F.; Nicot, F.; Sandres-Sauné, K.; Dubois, M.; Legrand-Abravanel, F.; Alric, L.; Peron, J.M.; Pasquier, C.; Izopet, J. Genetic diversity of HCV genotype 2 strains in south western France. J Med Virol 2007, 79, 26-34.

10. Markov, P.V.; van de Laar, T.J.; Thomas, X.V.; Aronson, S.J.; Weegink, C.J.; van den Berk, G.E.; Prins, M.; Pybus, O.G.; Schinkel, J. Colonial history and contemporary transmission shape the genetic diversity of hepatitis C virus genotype 2 in Amsterdam. Journal of virology 2012, 86, 7677-7687.

11. Haldeda, M.; Baume, J.; Tamalet, C.; Bizhga, M.; Colson, P. Hepatitis C virus genotypes in Tirana, Albania. International journal of infectious diseases : IJID : official publication of the International Society for Infectious Diseases 2014, 18, 90-93.

12. Kondili, L.A.; Çuko, L.; Chionne, P.; Candido, A.; Madonna, E.; Dentico, P.; Resuli, B.; Taliani, G.; Brunetto, M.R.; Rapicetta, M. Hepatitis B, C and Delta virus infections in Albanian patients with chronic liver disea se: evaluation of possible changes during the last 10 years. European journal of gastroenterology \& hepatology 2010, 22, 167-171, doi:10.1097/MEG.0b013e328330d410.

13. Petruzziello, A.; Loquercio, G.; Sabatino, R.; Balaban, D.V.; Ullah Khan, N.; Piccirillo, M.; Rodrigo, L.; di Capua, L.; Guzzo, A.; Labonia, F., et al. Prevalence of Hepatitis C virus genotypes in nine selected European countries: A systematic review. J Clin Lab Anal 2019, 33, e22876.

14. Doçi, P.; Ebranati, E.; Zehender, G.; Dragusha, E.; Fiaschi, L.; Carta, V.; Veo, C.; Bino, S.; Seferi, I.; Shkjezi, R. Prevalence of HCV virus genotypes in Albania. International Journal of Engineering Science 2017, 06, 59-63.

15. Ansaldi, F.; Bruzzone, B.; Salmaso, S.; Rota, M.C.; Durando, P.; Gasparini, R.; Icardi, G. Different seroprevalence and molecular epidemiology patterns of hepatitis C virus infection in Italy. J Med Virol 2005, 76, 327-332. 
16. Roffi, L.; Ricci, A.; Ogliari, C.; Scalori, A.; Minola, E.; Colloredo, G.; Donada, C.; Ceriani, R.; Rinaldi, G.; Paris, B., et al. HCV genotypes in Northern Italy: a survey of 1368 histologically proven chronic hepatitis $\mathrm{C}$ patients. Journal of hepatology 1998, 29, 701-706.

17. Dal Molin, G.; Ansaldi, F.; Biagi, C.; D'Agaro, P.; Comar, M.; Crocè, L.; Tiribelli, C.; Campello, C. Changing molecular epidemiology of hepatitis C virus infection in Northeast Italy. J Med Virol 2002, 68, 352-356.

18. Bortolotti, F.; Guido, M.; Zancan, L.; Gussetti, N. Long-term outcome of hepatitis C in children. Hepatology 2004, 39, 1455; author reply 1455-1456.

19. Guadagnino, V.; Stroffolini, T.; Rapicetta, M.; Costantino, A.; Kondili, L.A.; Menniti-Ippolito, F.; Caroleo, B.; Costa, C.; Griffo, G.; Loiacono, L., et al. Prevalence, risk factors, and genotype distribution of hepatitis $\mathrm{C}$ virus infection in the general population: a community-based survey in southern Italy. Hepatology 1997, 26, 1006-1011.

20. Matera, G.; Lamberti, A.; Quirino, A.; Focà, D.; Giancotti, A.; Barreca, G.S.; Guadagnino, V.; Liberto, M.C. Changes in the prevalence of hepatitis $\mathrm{C}$ virus (HCV) genotype 4 in Calabria, Southern Italy. Diagn Microbiol Infect Dis 2002, 42, 169-173.

21. Pistello, M.; Maggi, F.; Vatteroni, L.; Cecconi, N.; Panicucci, F.; Bresci, G.P.; Gambardella, L.; Taddei, M.; Bionda, A.; Tuoni, M., et al. Prevalence of hepatitis C virus genotypes in Italy. J Clin Microbiol 1994, 32, 232-234.

22. Cenci, M.; Massi, M.; Alderisio, M.; De Soccio, G.; Recchia, O. Prevalence of hepatitis C virus (HCV) genotypes and increase of type 4 in central Italy: an update and report of a new method of HCV genotyping. Anticancer Res $2007,27,1219-1222$.

23. Pontisso, P.; Ruvoletto, M.G.; Nicoletti, M.; Tisminetzky, S.; Gerotto, M.; Levrero, M.; Artini, M.; Baldi, M.; Ballardini, G.; Barbara, L., et al. Distribution of three major hepatitis C virus genotypes in Italy. A multicentre study of 495 patients with chronic hepatitis C. J Viral Hepat 1995, 2, 33-38.

24. De Paschale, M.; Casiraghi, M.A.; Biagiotti, S.; Rossi, U.; Zanetti, A.R. Association between neonatal blood microtransfusions in the 1960s and hepatitis C virus infection. Lancet 2000, 356, 1572-1573.

25. Esteban, J.I.; Sauleda, S.; Quer, J. The changing epidemiology of hepatitis C virus infection in Europe. Journal of hepatology 2008, 48, 148-162.

26. Markov, P.V.; Pepin, J.; Frost, E.; Deslandes, S.; Labbé, A.C.; Pybus, O.G. Phylogeography and molecular epidemiology of hepatitis C virus genotype 2 in Africa. The Journal of general virology 2009, 90, 2086-2096.

Purdy, M.A.; Forbi, J.C.; Sue, A.; Layden, J.E.; Switzer, W.M.; Opare-Sem, O.K.; Phillips, R.O.; Khudyakov, Y.E. A re-evaluation of the origin of hepatitis C virus genotype 2 in West Africa. The Journal of general virology 2015, 96, 2157-2164.

28. Ho, S.Y.; Saarma, U.; Barnett, R.; Haile, J.; Shapiro, B. The effect of inappropriate calibration: three case studies in molecular ecology. PLoS One 2008, 3, e1615.

29. Power, J.P.; Lawlor, E.; Davidson, F.; Holmes, E.C.; Yap, P.L.; Simmonds, P. Molecular epidemiology of an outbreak of infection with hepatitis C virus in recipients of anti-D immunoglobulin. Lancet 1995, 345, 1211-1213.

30. Pybus, O.G.; Charleston, M.A.; Gupta, S.; Rambaut, A.; Holmes, E.C.; Harvey, P.H. The epidemic behavior of the hepatitis C virus. Science 2001, 292, 2323-2325.

31. Peck, K.M.; Chan, C.H.; Tanaka, M.M. Connecting within-host dynamics to the rate of viral molecular evolution. Virus Evol 2015, 1, vev013. 
33. Salemi, M.; Lewis, M.; Egan, J.F.; Hall, W.W.; Desmyter, J.; Vandamme, A.M. Different population dynamics of human T cell lymphotropic virus type II in intravenous drug users compared with endemically infected tribes. Proc Natl Acad Sci U S A 1999, 96, 13253-13258.

34. Streicker, D.G.; Lemey, P.; Velasco-Villa, A.; Rupprecht, C.E. Rates of viral evolution are linked to host geography in bat rabies. PLoS Pathog 2012, 8, e1002720.

35. Hanada, K.; Suzuki, Y.; Gojobori, T. A large variation in the rates of synonymous substitution for RNA viruses and its relationship to a diversity of viral infection and transmission modes. Molecular biology and evolution 2004, 21, 1074-1080, doi:10.1093/molbev/msh109.

36. Gray, R.R.; Parker, J.; Lemey, P.; Salemi, M.; Katzourakis, A.; Pybus, O.G. The mode and tempo of hepatitis C virus evolution within and among hosts. BMC Evol Biol 2011, 11, 131.

37. Salemi, M.; Vandamme, A.M. Hepatitis C virus evolutionary patterns studied through analysis of full-genome sequences. J Mol Evol 2002, 54, 62-70.

38. Marascio, N.; Ciccozzi, M.; Equestre, M.; Lo Presti, A.; Costantino, A.; Cella, E.; Bruni, R.; Liberto, M.C.; Pisani, G.; Zicca, E., et al. Back to the origin of HCV 2c subtype and spreading to the Calabria region (Southern Italy) over the last two centuries: a phylogenetic study. Infection, genetics and evolution : journal of molecular epidemiology and evolutionary genetics in infectious diseases 2014, 26, 352-358.

39. Hundie, G.B.; Raj, V.S.; GebreMichael, D.; Pas, S.D.; Haagmans, B.L. Genetic diversity of hepatitis C virus in Ethiopia. PLoS One 2017, 12, e0179064.

40. Pybus, O.G.; Cochrane, A.; Holmes, E.C.; Simmonds, P. The hepatitis C virus epidemic among injecting drug users. Infection, genetics and evolution : journal of molecular epidemiology and evolutionary genetics in infectious diseases 2005, 5, 131-139.

41. Bonifazi, C.; Sabatino, D. Albanian migration to Italy: what official data and survey results can reveal. Journal of Ethnic and Migration Studies 2003, 29, 967-995, doi:10.1080/1369183032000171320.

Zehender, G.; Sorrentino, C.; Lai, A.; Ebranati, E.; Gabanelli, E.; Lo Presti, A.; Vujoševic, D.; Lauševic, D.; Terzić, D.; Shkjezi, R., et al. Reconstruction of the evolutionary dynamics of hepatitis $C$ virus subtypes in Montenegro and the Balkan region. Infection, genetics and evolution : journal of molecular epidemiology and evolutionary genetics in infectious diseases 2013, 17, 223-230.

43. Lu, L.; Nakano, T.; Smallwood, G.A.; Heffron, T.G.; Robertson, B.H.; Hagedorn, C.H. A refined long RT-PCR technique to amplify complete viral RNA genome sequences from clinical samples: application to a novel hepatitis C virus variant of genotype 6. Journal of virological methods 2005, 126, 139-148.

44. Strimmer, K.; von Haeseler, A. Likelihood-mapping: a simple method to visualize phylogenetic content of a sequence alignment. Proc Natl Acad Sci U S A 1997, 94, 6815-6819.

45. Schmidt, H.A.; Strimmer, K.; Vingron, M.; von Haeseler, A. TREE-PUZZLE: maximum likelihood phylogenetic analysis using quartets and parallel computing. Bioinformatics 2002, 18, 502-504.

46. Thompson, J.D.; Higgins, D.G.; Gibson, T.J. CLUSTAL W: improving the sensitivity of progressive multiple sequence alignment through sequence weighting, position-specific gap penalties and weight matrix choice. Nucleic Acids Res 1994, 22, 4673-4680.

47. Drummond, A.J.; Suchard, M.A.; Xie, D.; Rambaut, A. Bayesian phylogenetics with BEAUti and the BEAST 1.7. Molecular biology and evolution 2012, 29, 1969-1973.

48. Lemey, P.; Suchard, M.; Rambaut, A. Reconstructing the initial global spread of a human influenza pandemic: A Bayesian spatial-temporal model for the global spread of H1N1pdm. PLoS Curr 2009, 1, RRN1031.

49. Suchard, M.A.; Weiss, R.E.; Sinsheimer, J.S. Bayesian selection of continuous-time Markov chain evolutionary models. Molecular biology and evolution 2001, 18, 1001-1013. 
50. Kass, R.E.; Raftery, A.E. Bayes Factors. Journal of the American Statistical Association 1995, 90, 773-795, doi:10.2307/2291091.

51. Bielejec, F.; Baele, G.; Vrancken, B.; Suchard, M.A.; Rambaut, A.; Lemey, P. SpreaD3: Interactive Visualization of Spatiotemporal History and Trait Evolutionary Processes. Molecular biology and evolution 2016, 33, 2167-2169.

52. Stadler, T.; Kühnert, D.; Bonhoeffer, S.; Drummond, A.J. Birth-death skyline plot reveals temporal changes of epidemic spread in HIV and hepatitis C virus (HCV). Proc Natl Acad Sci U S A 2013, 110, 228-233. 